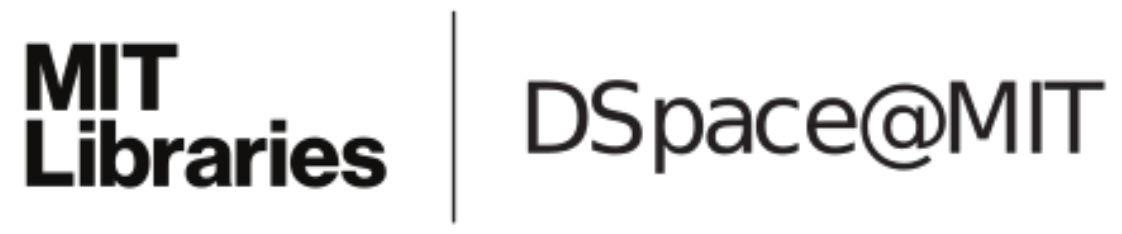

\author{
MIT Open Access Articles
}

Single-Mode and High-Speed 850nm MEMS-VCSEL

The MIT Faculty has made this article openly available. Please share how this access benefits you. Your story matters.

Citation: D. D. John et al, "Single-Mode and High-Speed 850nm MEMS-VCSEL," Advanced Solid State Lasers 2016, October-November 2016, Boston Massachusetts, United States, Optical Society of America, 2016. (C) 2016 Optical Society of America

As Published: http://dx.doi.org/10.1364/assl.2016.ath5a.2

Publisher: Optical Society of America

Persistent URL: https://hdl.handle.net/1721.1/121416

Version: Author's final manuscript: final author's manuscript post peer review, without publisher's formatting or copy editing

Terms of use: Creative Commons Attribution-Noncommercial-Share Alike 


\title{
Single-Mode and High-Speed 850nm MEMS-VCSEL
}

\author{
Demis D. John ${ }^{1}$, Byungkun Lee ${ }^{2}$, Benjamin Potsaid ${ }^{2,3}$, Amanda C. Kennedy ${ }^{1}$, Martin E. Robertson ${ }^{1}$, \\ Christopher B. Burgner ${ }^{1}$, Alex E. Cable ${ }^{3}$, James G. Fujimoto ${ }^{2}$, Vijaysekhar Jayaraman ${ }^{1}$ \\ ${ }^{I}$ Praevium Research Inc., 5385 Hollister Avenue, Santa Barbara, CA 93111, U.S.A. \\ ${ }^{2}$ Massachusetts Institute of Technology, Dept. of Electrical \& Computer Engineering, 50 Vassar St., Cambridge, MA 02139, U.S.A. \\ ${ }^{3}$ Thorlabs Inc., 56 Sparta Ave., Newton, NJ 07860, U.S.A. \\ Authore-mail address: demis@praevium.com
}

\begin{abstract}
We present an 850nm Vertical-Cavity Surface-Emitting Laser (VCSEL) with electrostatically-actuated top mirror. Developed to target the water-transmission window for ophthalmic imaging, the light source achieves $37.7 \mathrm{~nm}$ of tuning at $347 \mathrm{kHz}$ unamplified, and $5.4 \mathrm{~mW}$ amplified power.

OCIS codes: (140.3600) Tunable lasers; (140.7260) Vertical cavity surface emitting lasers
\end{abstract}

\section{Introduction}

Research on new light sources for the medical imaging technique known as Optical Coherence Tomography (OCT) has been very active in recent years. Prototype light sources previously relegated to the lab are being developed as commercial OCT swept-sources, often targeted to specific medical imaging applications. Ophthalmology, being one of the most significant OCT markets, has spurred much of this interest in new light sources.

The MEMS-Tunable VCSEL (Microelectromechanically-Tunable Vertical-Cavity Surface-Emitting Laser) has significant advantages for OCT due to the single-mode (both transverse \& longitudinal) and mode-hop free tuning, along with high-speed tuning at hundreds of $\mathrm{kHz}$ and long coherence lengths as compared with other popular OCT light sources [1-3]. The speed and tuning range of previously demonstrated $1310 \mathrm{~nm} \& 1050 \mathrm{~nm}$ tunable sources with $110 \mathrm{~nm} \& 100 \mathrm{~nm}$ optical tuning bandwidths, respectively [1,4], has enabled numerous advances in endoscopic \& ophthalmic imaging [5-7].

The wavelength of light sources for ophthalmic OCT is primarily chosen to target a region of the water absorption spectrum in which there is low optical loss. Our MEMS-VCSEL sources at 1050nm were chosen to target the low-loss 1060nm region in the water absorption spectrum, as shown in Figure 1.

A near-IR swept-source in the low-absorption $850 \mathrm{~nm}$ region may enable better resolution compared to $1050 \mathrm{~nm}$, in addition to the possible imaging-range benefit of swept-source OCT versus the more common spectral domain (broad-band source) OCT. These reasons have motivated the development of a new 850nm MEMS-VCSEL intended for ophthalmic OCT imaging that we present for the first time in this paper.

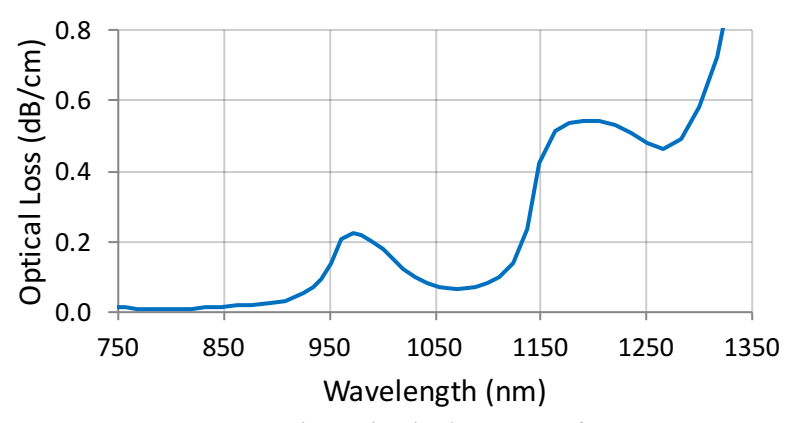

Fig. 1: Water Absorption in the near-IR from [8].

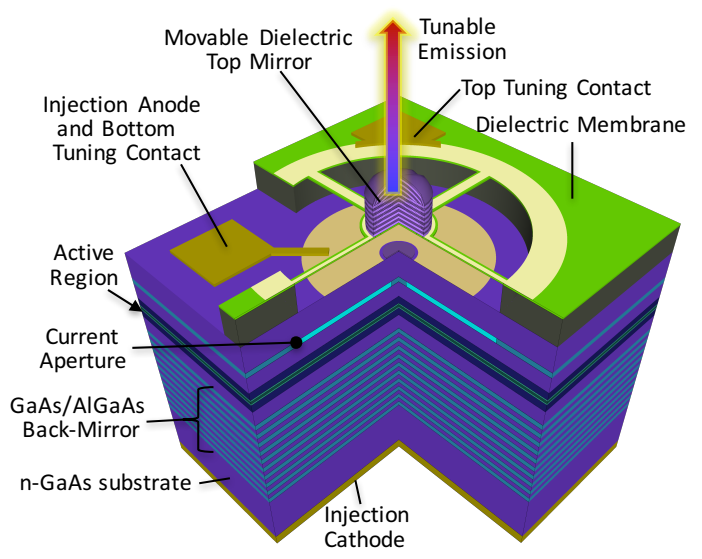

Fig. 2: Schematic of the $850 \mathrm{~nm}$ MEMS-VCSEL.

\section{Design}

The core MEMS wavelength-tuning component of previous devices is extensible to other wavelength ranges and the GaAs-based materials used in our 1050nm MEMS-VCSELs is easily extended into the $850 \mathrm{~nm}$ range. A P-i-N diode structure was grown with a standard GaAs/AlGaAs back mirror, as schematically shown in Figure 2 . Targeting single-mode operation, we fabricated devices with thermally-oxidized current apertures, formed by converting the as-grown AlAs current-aperture layer into $\mathrm{AlO}_{\mathrm{x}}$, leaving an unoxidized aperture diameter varying from $2-7 \mu \mathrm{m}$. A metal contact on the p-type GaAs surface of the semiconductor acts as the laser injection anode and 
funnels current through the unoxidized portion of the current aperture into AlGaAs quantum wells. Finally, current exits through the back mirror and n-GaAs substrate, to a laser injection cathode on the substrate underside.

The Injection Anode also acts as the MEMS bottom contact. Since the MEMS draws negligible current, it is possible to apply an AC or DC voltage between the MEMS bottom \& top contacts, constricting the air gap and tuning the cavity mode, while simultaneously applying a DC current to the laser injection contacts without the two signals interacting significantly. The top-half of the cavity was completed similarly to our previous devices described in [1] and [4].

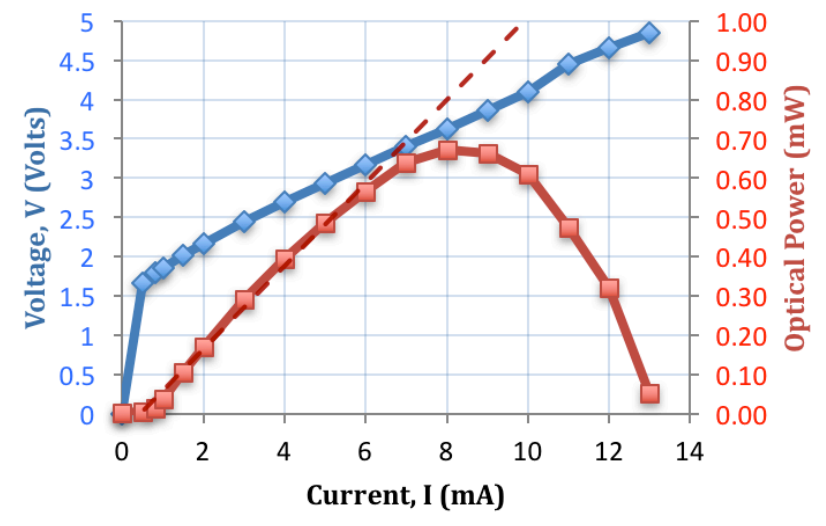

Fig. 3: L-I-V curves show a threshold laser current of $0.79 \mathrm{~mA}$ at a rest wavelength of $850.2 \mathrm{~nm}$, and a maximum optical power of $672 \mu \mathrm{W}$.

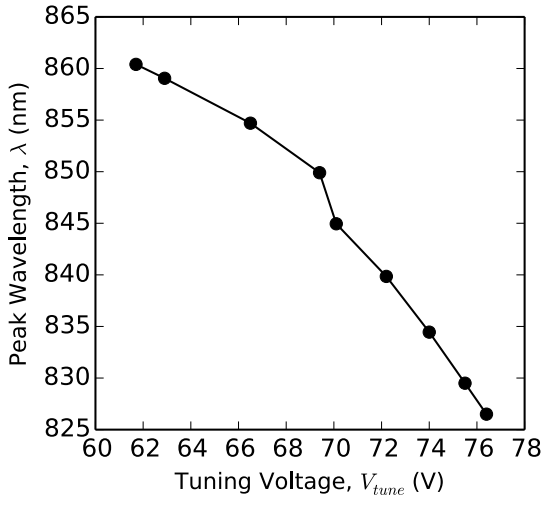

Fig. 4: Peak wavelength vs. Tuning Voltage

\section{Optical Testing Results}

Previous researchers have shown MEMS-VCSELs operating at $850 \mathrm{~nm}$ targeting optical communications $[9,10]$. The previous work achieved $19 \mathrm{~nm}$, and later $37 \mathrm{~nm}$, of tuning at a tuning speed of $0.70 \mathrm{kHz}$ with thermal tuning of the MEMS. These devices lased in multiple lateral modes, with about $0.4 \mathrm{~mW}$ of output power. Targeting OCT instead, we have applied our electrostatic MEMS technology to address the requirements of high-tuning speed, single-mode operation and wide bandwidth. In addition, higher output power is required for ophthalmic imaging.

First-generation devices exhibit a thermal rollover at $672 \mu \mathrm{W}$, as shown in Fig. 3. The devices are single-lateralmode up to $6 \mathrm{~mA}$ with $\geq 38 \mathrm{~dB}$ SMSR (side-mode suppression ratio), above which a second-order lateral mode begins to lase. Figures $4 \& 5$ show the tuning range at $4 \mathrm{~mA}$ laser current, with $36.0 \mathrm{~nm}$ of continuously-swept tuning (-6dB) at $429 \mathrm{kHz}$ (dark green) in a single longitudinal mode. By reducing the airgap further, the device switches into a second longitudinal mode, as shown in the double-peaked $61.7 \mathrm{~V}$ discrete spectrum of Fig. 5. Electrostatic softening reduces the stiffness of the membrane and decreases the resonance frequency for this tuning range, resulting in $37.7 \mathrm{~nm}$ of continuous single-mode tuning at $347 \mathrm{kHz}$ (light green) for a $6 \mathrm{~mA}$ laser current. The spectra in Fig. 5 were acquired with $200 \mu \mathrm{m}$-core multi-mode optical fibers to capture any lateral modes, while a single-mode fiber (SMF) coupled device showed a slightly improved SMSR of 43dB. All devices operate in a single polarization.

Mode profiles were acquired at the rest-wavelengths $\left(V_{T U N E}=0.0 \mathrm{~V}\right)$ of various unpackaged devices in the same production run with a Thorlabs M2MS-BP209IR measurement system, the results of which are shown in Fig. 7.

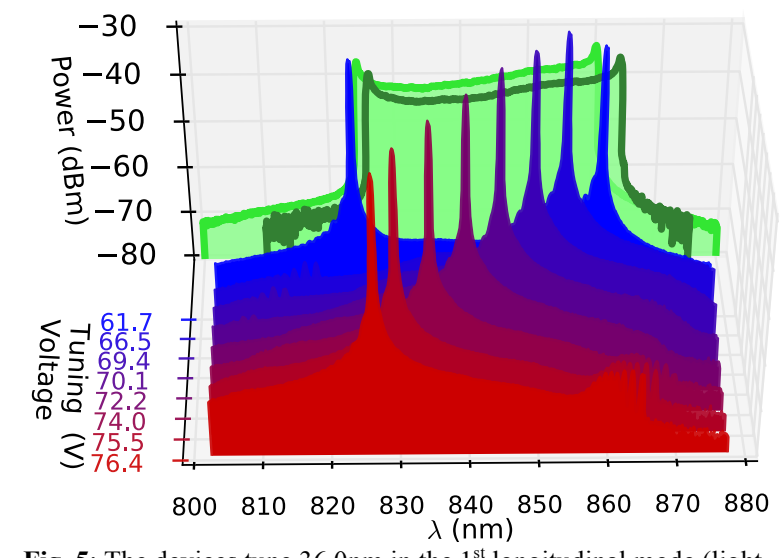

Fig. 5: The devices tune $36.0 \mathrm{~nm}$ in the $1^{\text {st }}$ longitudinal mode (light green), and $37.7 \mathrm{~nm}$ in the $2^{\text {nd }}$ longitudinal mode (dark green) at $4 \mathrm{~mA} \&$ $6 \mathrm{~mA}$ laser currents, respectively.

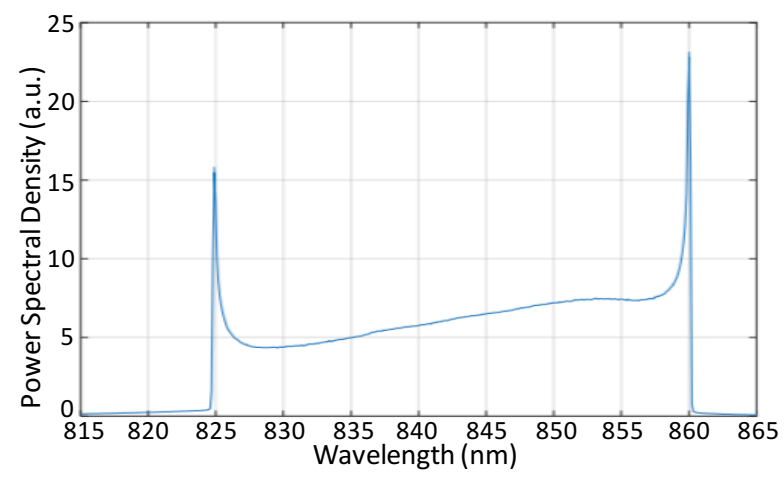

Fig. 6: Spectrum of $400 \mathrm{kHz}$ dynamic tuning amplified by an SOA, with $5.38 \mathrm{~mW}$ optical power output at $96 \mathrm{~mA}$ and $53 \%$ pulse-width modulated duty cycle. 

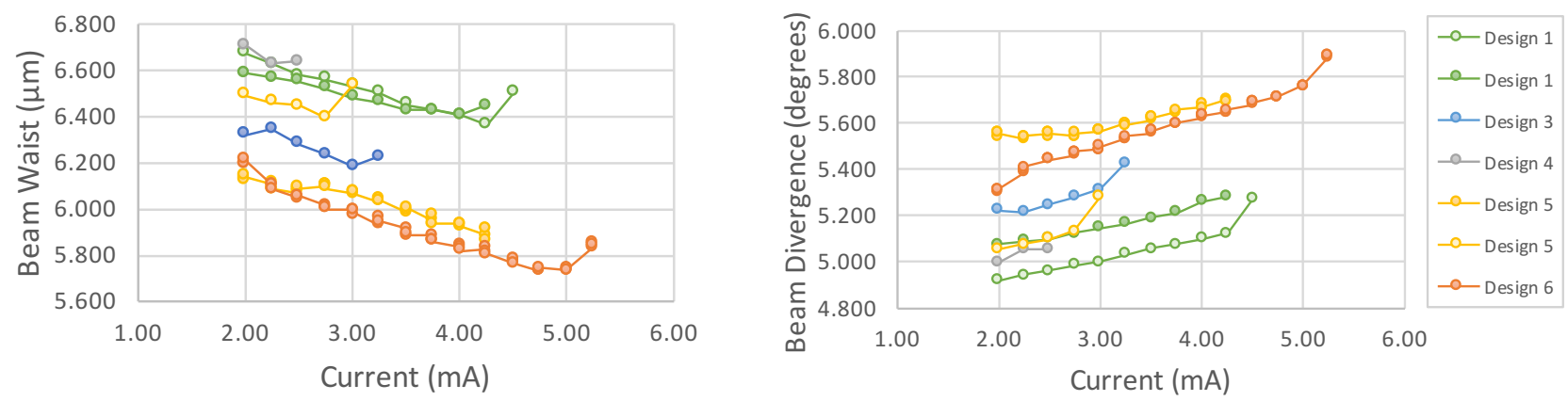

Fig. 7: Beam Waist (diameter) and Beam Divergence (half-angle) versus Laser Current, for various devices from the same run.

In Fig. 7, each design represents a current-aperture size variation of nominally $0.25 \mu \mathrm{m}$, with Design 1 being the largest, although the actual aperture size has some deviation from the nominal design. The sudden uptick at higher currents corresponds to a reduced beam measurement quality, likely due to higher-order lateral modes. Beam waist diameters vary between $5.7-6.7 \mu \mathrm{m}$, with decreasing waist versus laser drive current, while beam divergence halfangles range from $4.9-5.9^{\circ}$ with increasing divergence versus laser current.

The optical power must be amplified in order to take full advantage of the OCT optical power limits. Although few commercial wide-band SOAs (semiconductor optical amplifiers) are available in the $850 \mathrm{~nm}$ range, our device is well-matched to the Superlum SOA-352. A MEMS-VCSEL packaged with SMF was amplified with the aforementioned SOA, with AC Photonics isolators on either side of the SOA, resulting in an amplified optical power of $5.38 \mathrm{~mW}$ for a $400 \mathrm{kHz}$ sweep. Figure 6 shows the amplified spectrum with a $96 \mathrm{~mA}$ SOA current which is pulsewidth modulated with a $53 \%$ duty cycle to output only a single sweep direction as a light source suitable for OCT.

\section{Conclusions}

We have shown an $850 \mathrm{~nm}$ MEMS-VCSEL with single-mode tuning over $37.7 \mathrm{~nm}$ at high sweep speeds of 350 $430 \mathrm{kHz}$, with up to $670 \mu \mathrm{W}$ of unamplified optical power, and a fiber-coupled side-mode suppression ratio of $43 \mathrm{~dB}$. When utilized with a matched amplifier, we achieved average optical powers of $5.38 \mathrm{~mW}$ at $400 \mathrm{kHz}$ sweep rates in a single-scan direction.

This new $850 \mathrm{~nm}$ swept-source will be applied to ophthalmic OCT imaging in a future publication.

\section{References}

[1] Jayaraman, V., Jiang, J., Li, H., Heim, P., Cole, G., Ben Potsaid, Fujimoto, J. G., Cable, A., "OCT Imaging up to 760Khz Axial Scan Rate Using Single-Mode 1310nm MEMs-Tunable VCSELs with >100nm Tuning Range," in Proc. of CLEO:2011 - Laser Applications to Photonic Applications, Paper PDPB2, 2011.

[2] Grulkowski, I., Liu, J. J., Potsaid, B., Jayaraman, V., Lu, C. D., Jiang, J., Cable, A. E., Duker, J. S. and Fujimoto, J. G., "Retinal, anterior segment and full eye imaging using ultrahigh speed swept source OCT with vertical-cavity surface emitting lasers," Biomedical Optics Express, 3(11), 2733-2751, 2012.

[3] Lu, C. D., Kraus, Martin F, Potsaid, B., Liu, J. J., Choi, W., Jayaraman, V., Cable, A. E., Hornegger, J., Duker, J. S., Fujimoto, J. G., "Handheld ultrahigh speed swept source optical coherence tomography instrument using a MEMS scanning mirror," Biomedical Optics Express, 5(1), 293-311, 2014

[4] Jayaraman, V., Cole, G. D., Robertson, M., Burgner, C., John, D. D., Uddin, A., \& Cable, A., "Rapidly swept, ultra-widely-tunable $1060 \mathrm{~nm}$ MEMS-VCSELs," Electronics Letters, 48(21), 2012.

[5] Tsai, T.-H., Potsaid, B., Tao, Y. K., Jayaraman, V., Jiang, J., Heim, P. J. S., Kraus, M. F., Zhou, C., Hornegger, J., Mashimo, H., Cable, A. E., Fujimoto, J. G., "Ultrahigh speed endoscopic optical coherence tomography using micromotor imaging catheter and VCSEL technology," Biomedical Optics Express, 4(7), 1119-1132, 2013.

[6] Choi, W., Mohler, K. J., Potsaid, B., Lu, C. D., Liu, J. J., Jayaraman, V., Cable, A. E., Duker, J. S., Huber, R., Fujimoto, J. G., "Choriocapillaris and Choroidal Microvasculature Imaging with Ultrahigh Speed OCT Angiography," PloS One, 8(12), e81499, 2013.

[7] D. D. John, C. Burgner, B. Potsaid, M. Robertson, B. Lee, W. J. Choi, A. Cable, J. Fujimoto, and V. Jayaraman, "Wideband ElectricallyPumped $1050 \mathrm{~nm}$ MEMS-Tunable VCSEL for Ophthalmic Imaging," Journal of Lightwave Technology, vol. 33, no. 16, pp. 3461-3468, Feb. 2015.

[8] K. F. Palmer and D. Williams, "Optical properties of water in the near infrared,” J. Opt. Soc. Am., vol. 64, no. 8, p. $1107,1974$.

[9] H. A. Davani, C. Grasse, B. Kogel, P. Westbergh, C. Gierl, K. Zogal, S. Jatta, G. Bohm, T. Gruendl, P. Meissner, A. Larsson, and M.-C. Amann, "Widely tunable high-speed bulk-micromachined short-wavelength MEMS-VCSEL," presented at the IEEE International Semiconductor Laser Conference, paper MB4, 2010.

[10] H. A. Davani, C. Grasse, B. Kogel, C. Gierl, K. Zogal, T. Gründl, P. Westbergh, S. Jatta, G. Bohm, P. Meissner, A. Larsson, and M. C. Amann, "Widely electro thermal tunable bulk-Micromachined MEMS-VCSEL operating around 850nm," presented at the 2011 International Quantum Electronics Conference (IQEC) and Conference on Lasers and Electro-Optics (CLEO) Pacific Rim, Sydney, Australia, pp. 32-34, 2011.

\section{Acknowledgements}

Funding was provided by NEI SBIR grant R44EY022864-03 and Thorlabs Inc. The content of this publication is solely the responsibility of the authors and does not necessarily represent the views of the National Eye Institute of the National Institutes of Health. 